DR. SUBHAJIT CHAKRAVORTY (Orcid ID : 0000-0001-5225-3489)

3

4

7

Article type : Original Research Article
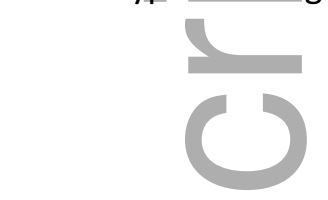

\title{
Cognitive Behavior
}

Cognitive Behavioral Therapy for Insomnia in Alcohol Dependent Veterans: A Randomized,

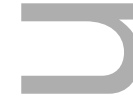

\section{Controlled Pilot Study}

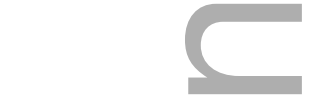

Subhajit Chakravorty MD ${ }^{1,2}$, Knashawn H. Morales ScD², J. Todd Arnedt PhD², Michael L. Perlis

12
$\mathrm{PhD}^{2}$, David W. Oslin $\mathrm{MD}^{1,2}$, James C. Findley $\mathrm{PhD}^{4}$, Henry R. Kranzler MD ${ }^{1,2}$

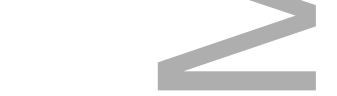

${ }^{1}$ Cpl. Michael J. Crescenz VA Medical Center, Philadelphia, PA; ${ }^{2}$ Center for Studies of Addiction, Perelman School of Medicine, Philadelphia, PA; ${ }^{3}$ University of Michigan Medical School, Ann Arbor, MI; ${ }^{4}$ Penn Sleep Center, Perelman School of Medicine, Philadelphia, PA

7

(1)

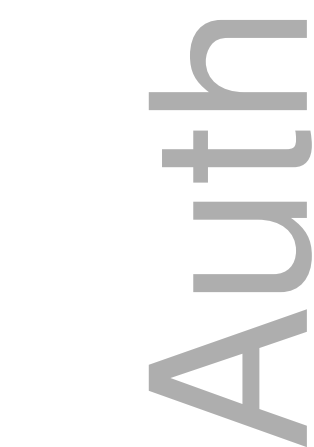 \\ 21 Corresponding Author:}

This is the author manuscript accepted for publication and has undergone full peer review but has not been through the copyediting, typesetting, pagination and proofreading process, which may lead to differences between this version and the Version of Record. Please cite this article as doi: 10.1111/ACER.14030

This article is protected by copyright. All rights reserved 
23 Subhajit Chakravorty, M.D.

24 MIRECC, 2nd Floor, Postal Code 116

25 Corporal Michael J. Crescenz VA Medical Center

263900 Woodland Avenue

27 Philadelphia, PA 19104

28 Telephone:(215)-823-5800 x 6509

29 Fax: (215)-823-4123

30 Email : Subhajit.Chakravorty@uphs.upenn.edu

31

32 Support: This study was funded by the Competitive Pilot Project Fund of the Veterans 33 Integrated Service Network 4 and supported by VA grant IK2CX000855 (S.C.) and NIH grants 34 R01 AG041783 (to M.L.P.), R56 AG050620 (to M.L.P.); and R01 AA023192 and R01 AA021164 35 (to H.R.K.).

36 Word count: 3973.

37 Numbers of tables and figures: 6 ( 2 tables +4 figures)

38 clinicaltrials.gov identifier NCT01603381.

39

40

41 Abstract

42 Background: Insomnia is highly prevalent in individuals recovering from Alcohol Dependence 43 (AD) and increases their risk of relapse. Two studies evaluating Cognitive Behavioral Therapy for 
Insomnia (CBT-I) have demonstrated its efficacy in non-Veterans recovering from AD. The aim of this study was to extend these findings in an 8-week trial of CBT-I in Veterans.

Methods: Veterans recovering from AD were randomly assigned to 8 weeks of treatment with CBT-I $(N=11)$ or a Monitor-Only (MO $[n=11])$ condition and were evaluated $3(N=21 / 22)$ and 6 months post-treatment $(\mathrm{N}=18 / 22)$. The primary outcome measure was the Insomnia Severity Index (ISI) score. Secondary outcome measures were sleep diary measures, percent days abstinent (PDA), and scores on the Dysfunctional Beliefs and Attitudes About Sleep scale (DBAS), Sleep Hygiene Index (SHI), Penn Alcohol Craving Scale (PACS), Quick Inventory of Depressive Symptoms (QIDS), State-Trait Anxiety Inventory-Trait (STAI-T) scale and SF-12. Mixed effects regression models, adjusted for race, evaluated differences in outcomes between the groups over a 6-month period (clinicaltrials.gov identifier=NCT01603381).

Results: Subjects were male, aged 54.5 (SD=6.9) years, and had 26.4 (SD=26.3) days of abstinence before their baseline evaluation. CBT-I produced a significantly greater improvement in model-based estimates than MO (mean change at 6 months compared to their baseline) for ISI, sleep latency from a daily sleep diary, DBAS mean score, and SHI total score. PDA and QIDS improved over time, but there was no difference between the groups. PACS, STAI-T or SF-12 scale did not show any improvement from their baseline scores.

Conclusions: CBT-I treatment demonstrated substantial efficacy in reducing insomnia, associated negative cognitions and improving sleep hygiene in Veterans during early recovery, though it did not reduce drinking behavior.

\section{Introduction}

Insomnia occurs in about two-thirds of recovering patients with alcohol dependence (AD), an estimated prevalence that is up to 6.5 times that of the general population (Chakravorty et al., 2016). Comorbid insomnia is linked with an increased risk of relapse during recovery from $A D$, as well as suicidal behavior, daytime dysfunction, and decreased well-being (Chakravorty et al., 2016). 
Prior studies have investigated pharmacologic and behavioral treatments for insomnia in AD.

71 Treatment with medications such as gabapentin, trazodone, and quetiapine has not

72 consistently been shown to be beneficial in patients with these co-occurring disorders

73 (Chakravorty et al., 2016). In contrast, in this population, the efficacy of behavioral treatments

74 such as progressive muscle relaxation and cognitive behavior therapy for insomnia (CBT-I) is well demonstrated. CBT-I, a multicomponent treatment, consists of both behavioral (e.g., sleep restriction, stimulus control) and cognitive (e.g., cognitive restructuring) strategies, which target factors that perpetuate insomnia over time. Two randomized trials evaluated the efficacy of CBT-I in patients with AD. Currie and colleagues (2004) compared CBT-I to two other conditions - a self-help condition (reading a manual along with telephone support sessions) and a waitlist control (Currie et al., 2004)-in 60 community-dwelling individuals with AD. Subjects treated with CBT-I and the self-help condition demonstrated significant improvements in sleep continuity relative to pre-treatment and greater improvements than a waitlist control, improvements that were maintained for 6 months after treatment. They found no effect of

CBT-I on alcohol use. Arnedt and colleagues (2011) conducted a randomized controlled trial (RCT) of an 8-session version of CBT-I ( $N=9)$ or a behavioral placebo control $(\mathrm{N}=8)$ (Arnedt et al., 2011). CBT-1-treated subjects showed significantly greater improvements in self-reported Sleep Efficiency (SE) and Wake After Sleep Onset (WASO) than the control group, but no difference in alcohol consumption. Thus, although both studies showed that CBT-I improved insomnia, neither found that it reduced alcohol consumption.

While CBT-1 may be efficacious for insomnia among community-dwelling patients with AD, it has not been evaluated in Veterans with $A D$, a population with high prevalence rates of both insomnia and AD (Tsai et al., 2014, Kelsall et al., 2015, Seal et al., 2011, Alexander et al., 2016, Martin et al., 2017). Further, if CBT-I improves insomnia, does it also improve alcohol abstinence, as insomnia is a risk factor for relapse (Chakravorty et al., 2016)? The primary aim of this pilot study was to evaluate the efficacy of an 8-week CBT-I treatment for improving insomnia and at 3- and 6-month post-treatment follow-up in Veterans with AD during early recovery. Secondary aims of the study were to compare CBT-I to a Monitor-Only (MO) control group on alcohol-related outcomes and to assess the effect of CBT-I treatment on daytime 
functioning measures such as depressive symptoms, anxiety symptoms, and self-reported wellbeing.

\section{Materials and Methods}

We conducted an 8-week, randomized, parallel-group trial of CBT-I in 22 individuals. The study was conducted at the Cpl. Michael J Crescenz VA Medical Center (CMCVAMC) in Philadelphia, PA. The CMCVAMC Institutional Review Board approved the study and all subjects gave informed consent prior to participating in the study. Upon successful completion of screening, prospective subjects were randomly assigned using the Research Randomizer software (Urbaniak and Plous, 2013) to receive the active treatment or to a Monitor-Only arm (MO) and followed up in-person for 8 consecutive weeks. They returned for follow-up visits 3 and 6 months later.

Subjects. Subjects were recruited using flyers posted within the medical center or as direct referrals from their care providers in addiction psychiatry, general psychiatry, and primary care clinics. Subjects were included if they were: Veterans aged 18-65 years; reported insomnia symptoms, as assessed by a score of $\geq 8$ on the Insomnia Severity Index; had $<1$ year of abstinence from risky drinking, a past-year diagnosis of alcohol dependence as determined by the MINI (Sheehan et al., 1998), no significant evidence of alcohol withdrawal [Clinical Institute Withdrawal Assessment (CIWA) scale score $\leq 8$ (Sullivan et al., 1989)], $\geq 3$ consecutive days of abstinence from alcohol prior to a portable home sleep apnea test (HST); and were capable of communicating in English and giving written informed consent.

Subjects were excluded if they had a past-year diagnosis of a drug use disorder other than nicotine or cannabis, current unstable or serious psychiatric conditions (e.g., schizophrenia, bipolar disorder), unstable or serious medical illness, chronic pain leading to insomnia, evidence of severe cognitive impairment on the Blessed Orientation-Memory-Concentration test (Blessed et al., 1968), or untreated, moderate-to-severe obstructive sleep apnea (OSA) diagnosed by HST or non-adherence with positive airway pressure (PAP) treatment, defined as 
PAP use $<60 \%$ of the days with use $\leq 4$ hours per night on the nights they used the device, based on their objective PAP compliance data.

Procedures. Prospective subjects underwent screening over a 2-4 week period, during which time staff comprehensively assessed their sleep, medical, and psychiatric disorders, which included an HST and clinical evaluation for sleep-related disorders. Subjects were required to be totally abstinent from alcohol for $\geq 3$ consecutive days prior to the HST. Following screening, subjects entered an 8-week treatment phase with weekly visits to the research clinic. They were followed up at 3 and 6 months post-treatment to evaluate changes in sleep, drinking, and daytime outcomes. All questionnaires were completed during the clinic visit, except sleep diaries, which were filled out at home.

Treatments. Cognitive Behavioral Therapy for Insomnia (CBT-I): The CBT-I treatment was based on the published treatment manual CBT-I (Perlis et al., 2005) and established procedures at the Penn Behavioral Sleep Medicine clinic. Eleven subjects met individually with the study clinician (JF), a certified behavioral sleep medicine provider, for CBT-I. Session 1 served as an orientation session and the initiation of Sleep Restriction Therapy (SRT) (Spielman et al., 1987a, Spielman et al., 1987b) and Stimulus Control therapy (Bootzin and Perlis, 2011, Bootzin, 1972, Bootzin, 1984). Sleep restriction therapy required that subjects limit their time in bed to an amount similar to their average total sleep time. Subsequently, the provider gradually increased time in bed in 15-30 minute increments to the targeted sleep duration while ensuring that $85-90 \%$ of the time in bed was spent sleeping. The sleep opportunity was decreased by $15-30$ minutes if the time spent sleeping did not reach $85 \%$ or decreased below $85 \%$. Stimulus control instructions limit the amount of time one may spend in the bedroom while awake and the kind of behaviors one may engage in while in the bed or the bedroom, in order to strengthen the relationship between the bed or bedroom and consolidated sleep, i.e., with the subject lying down to sleep only when sleepy and avoiding the bed for anything other than sleep or sexual activity. Sessions 2 covered Sleep Hygiene (Stepanski and Wyatt, 2003, Zarcone, 1989, Posner and Gehrman, 2011), which addresses behaviors that influence sleep quality and quantity, such as arising at the same time every day; avoiding alcohol, especially in the evenings; and avoiding 
daytime naps. Sessions 4-7 were dedicated to a titration of time in bed and ensuring patient adherence. Session 4 also delivered a specific form of cognitive therapy, which was introduced initially in Session 3 (as a request to the patient to monitor his maladaptive cognitions for that week). The Cognitive Therapy was modeled on Barlow's approach to the cognitive restructuring of catastrophic thinking as it occurs with panic disorder (Barlow, 1992). This approach was adapted to address catastrophic thinking in relation to insomnia, such as "I will never be able to sleep on my own, ever again." The final session (session 8) focused on insomnia relapse prevention strategies. Adherence with CBT-I was assessed by calculating the difference between the prescribed Time-in-Bed and the reported Time-in-Bed on the sleep diaries. Deviations $\geq 105$ min each week were recoded into a dichotomous measure with subjects being considered compliant or noncompliant for that specified week (Perlis et al., 2004). Standard Care Monitor-Only Condition (M.O.): The eleven subjects in this condition were seen weekly by the study coordinator to complete and review assessments of their sleep, alcohol use, and daytime functioning. The review was interrogative in nature and no directed form of therapy was provided to help with sleep or reduce alcohol use, similar to other CBT-I studies that have used this control condition (Jungquist et al., 2010). As with the CBT-I sessions, the duration of the initial MO session was 45 minutes and each of the follow-up sessions was 30 minutes. MO subjects continued to receive ongoing medical and or mental health care. This condition controlled for the effects of observation and self-monitoring (Frank and Kaul, 1978, McCarney et al., 2007). Group therapy provided to Veterans at CMCVAMC for substance use disorders: The primary psychosocial treatment for addictive disorders at CMCVAMC is group therapy. It is grounded in the principles of 12-step meetings and Motivational Enhancement Therapy and modified by individual clinicians using either relapse prevention techniques or behavioral therapies (by employing techniques from Rational Emotive Behavioral Therapy and Cognitive Behavioral Therapy). No sleep-related intervention is employed in these groups but the importance of having a good sleep pattern is encouraged in one of the sessions to help prevent relapse.

Assessments. 
Sleep.

a) Insomnia Severity Index (ISI) (Bastien et al., 2001) is a 7-item, self-report questionnaire that yields a global score from 0-28, with higher scores representing greater insomnia severity. The ISI was the primary outcome measure in this study and was recorded every 2 weeks during treatment and at the post-treatment assessment visits;

b) Subjects completed the Perelman School of Medicine version of the daily sleep diary to assess subjective sleep variables at every treatment visit and at the 3- and 6-month post-treatment visits. The sleep diary variables used in this study included Sleep Latency (SL, time in minutes required to fall asleep initially); Wake After Sleep Onset time (WASO, duration of wakefulness during the night in minutes), Number of Awakenings (NAW, frequency of awakenings each night); and Total Sleep Time (TST, total time of sleep each night, in minutes).

c) Dysfunctional Beliefs and Attitudes About Sleep Scale (DBAS) (Morin et al., 2007). This 16-item questionnaire was completed at all assessment visits to evaluate unhelpful sleep-related cognitions.

d) Sleep Hygiene Index (SHI) (Mastin et al., 2006). This 13-item, patient-reported questionnaire assessed sleep hygiene behaviors at all assessment visits.

e) Epworth Sleepiness Scale scores (ESS) (Johns, 1991). This 8-item self-report measure evaluated daytime sleepiness at all assessment visits.

Alcohol Consumption.

a) Time Line Follow-back interview (Sobel and Sobell, 1992) was used to assess the percent days abstinent (PDA) for the 90 days during the baseline screening phase, during the 8week treatment phase, and at the 3- and 6-month follow-up visits. Heavy drinking was defined as the consumption of $\geq 5$ alcoholic drinks in a day.

b) Penn Alcohol Craving Scale (Flannery et al., 1999), a 5-item, self-report measure, was used to evaluate alcohol craving over the preceding 7 days at all assessment visits.

Daytime functioning.

This article is protected by copyright. All rights reserved 
a) Quick Inventory of Depressive Symptoms (QIDS) (Rush et al., 2003). This 16-item selfreport instrument assessed depressive symptoms at all assessment visits.

b) Trait sub-scale from the State-Trait Anxiety Inventory (STAI) (Spielberger et al., 1970). This 40 -item subject-rated scale was used to evaluate anxiety symptoms at all assessment visits.

c) Short Form 12-item (SF-12) (Ware et al., 1996) inquired about physical and mental wellbeing with the Physical and Mental Composite Scores (PCS and MCS), at baseline, weeks 4 and 8 of the treatment phase, and at 3- and 6-month post-treatment follow-up visits.

Statistical Analysis. We assessed baseline demographics and other characteristics across the treatment groups using a Wilcoxon Sign-rank test, t-test, chi-square or Fisher's test, as appropriate. Because the treatment groups were not balanced on self-reported race it was treated as a confounding variable in the analysis of longitudinal data. We used the intent-totreat principle to analyze the data. Linear mixed effects regression models employing maximum likelihood estimation were used to compare the outcome trajectory by treatment conditions across 10 time points ( 8 weekly treatment visits and 2 post-treatment follow-up visits). Exceptions to this rule were made with the ISI score, which was evaluated during 7 visits (baseline, treatment visits 2, 4, 6 and 8 and the two follow-up visits), and the PCS and MCS scores of the SF-12 scale, which was completed during 5 visits (treatment weeks 1, 4, and 8 and the two follow-up visits). Each model considered categorical, continuous, and quadratic trends over time and treatment by time interactions. We evaluated each of the models for the appropriate variance-covariance matrix structure of random effects. The final models for each variable were selected for the most parsimonious Bayesian Information Criterion score. The treatment effect was measured by the time-by-group interaction, where a statistically significant interaction indicated a difference in the outcome over time by group. Results are presented as scores of the model-estimated change from baseline along with their 95\% confidence intervals. Remission from insomnia was defined by a subject-specific, modelestimated ISI total score $<8$. Treatment response was defined as a decrease of $>8$ points in the ISI total score (Morin et al., 2011). 
A Generalized Estimating Equation was used to model the days abstinent from alcohol over time across treatment groups using a natural log link function and an independent within-group correlation structure, considering the Poisson distribution of PDA. The four time-segments used in this analysis were pretreatment (90 days), treatment (56 days), and 3- and 6-month posttreatment periods (each 90 days). We used the predicted value for each outcome generated from the longitudinal analysis to compute the difference scores and generate graphs, except in the evaluation of effect sizes (for ISI). The within-group effect sizes from baseline were assessed using the mean and standard deviation of the change score from baseline. Here, values of 0.20, 0.50, and 0.80 represent small, moderate, and large effect sizes, respectively (Friedmann et al., 2008). We used Stata 13.0 IC (StataCorp, 2011) to conduct statistical analysis and Stata 15.0 to create the graphs as Stata 13.0 was no longer available.

\section{Results}

Subjects. Twenty-four of 109 prospective subjects were eligible for the study, of whom two opted not to participate before randomization; see Figure 1 for a CONSORT flow chart. We randomly assigned 22 subjects to the CBT-I $(\mathrm{N}=11)$ or Monitor-Only (MO $[\mathrm{n}=11])$ condition. Sixteen of the 22 subjects were receiving psychosocial treatment for their AD, including 7 of 11 subjects in the CBT-I arm and 9 of 11 subjects in the MO arm, ( $p=N S$, not statistically significant). All 22 subjects completed the 8-week treatment period and 21 subjects completed the 3-month and 18 completed the 6-month post-treatment follow-up visits. During their study participation, 13 subjects relapsed to alcohol use (7 in the CBT-I arm and 6 in the MO arm, $\mathrm{p}=\mathrm{NS}$ ). Four subjects dropped out during the post-treatment follow-up period (2 in the CBT-I arm and 2 in the MO arm, NS). A comparison of subjects who dropped out of the study with those who completed it showed no difference on treatment allocation, pretreatment PDA, age, race, marital status, education or baseline ISI scores (p's all NS).

Baseline Demographics and Clinical Measures (Table 1). On average, subjects were middleaged, male, and African American (AA). The groups differed only on race, as the MO group was comprised entirely of AAs, while only $45 \%$ of the CBT-I arm was $(p=0.01)$. On average, subjects 
initially reported insomnia of moderate severity at baseline, with a mean sleep latency of 51 minutes. They were abstinent on about $26 \%$ of days during the 90 -day pretreatment period and reported heavy drinking on $80 \%$ of drinking days. There was no baseline treatment-group difference on the use of alcohol as a sleep aid.

Sleep Outcomes. 1) Insomnia Severity Index. Both groups showed a decrease in insomnia scores. However, at the end of treatment, there was a greater reduction in the ISI total score in the CBT-I group than the MO group, a difference that persisted at the 3-and 6-month follow-up visits (see Table 2 and Figure 2). When compared to their baseline values, the within-group magnitude of improvement at the end of treatment, and at the 3-month and 6-month followup visits, were 1.9, 2.2 and 1.9 for the CBT-I group, compared to $0.4,0.5$ and 0.4 for the MO group. All CBT-I treated subjects were remitted from their insomnia at week 8 , an effect that was maintained until the 6-month post-treatment follow-up visit. In contrast, none of the subjects in the $\mathrm{MO}$ arm were in remission from insomnia at the end of treatment or at the posttreatment follow-up visits; 2) Sleep diary variables. i) In a quadratic model, there was a treatment effect on SL, such that it initially decreased in the CBT-I group, but then increased in the post-treatment phase while remaining lower than in the $\mathrm{MO}$ at the 6-month follow-up visit (Figure 3). Exclusion of an outlier in the CBT-I group did not change the results; ii) WASO, TST and NAW - there was no treatment effect (Supplementary Figure 2); 3) Dysfunctional Beliefs and Attitudes About Sleep scale. There was a significant treatment effect, such that CBT-I subjects reported a lower DBAS mean score than the MO subjects (Figure 4). 4) Sleep Hygiene Index. There was a significant treatment effect, such that the CBT-I group reported a greater decrease in SHS total score over time, (Supplementary Figure 1). 5) Epworth Sleepiness Scale. Although there was not a significant effect of the intervention or time, there was a nonsignificant Treatment $x$ Time interaction effect $(p=0.05)$. 6) Treatment adherence with CBT-I. Only one subject met criteria for non-adherence, which occurred at visits $3,4,6$, and 7 and at the post-treatment follow-up visits.

Alcohol Consumption. 1) Percent Days Abstinent (PDA). At the beginning of treatment, 21 subjects were abstinent for all days in the preceding week; 1 subject in the MO arm had one 
drink on a single day. Our longitudinal assessment of PDA indicated that both groups improved over time, with no between-group difference (Supplemental Figure 3). During the 8 weeks of treatment, 18 subjects reported remaining abstinent from alcohol (10 in the CBT-I arm and 8 in the $\mathrm{MO}$ arm). At the 3-month follow-up visit, 14 of these 18 subjects reported remaining abstinent from alcohol ( 8 in the CBT-I arm and 6 in the $M O$ arm, $\chi^{2}=0.38, p=0.50$ ). Of these 14 subjects, 8 subjects remained abstinent at the 6-month follow-up visit (4 subjects in each treatment group). The subjects who relapsed during the study frequently drank heavily at those times and all received concomitant psychosocial treatment to reduce their drinking. Subjects who relapsed to drinking were not differentiated from the other participants on age, years of education, marital status, race, baseline PDA or ISI score, or treatment arm, or whether they received psychosocial treatment for AD. 2) Alcohol craving (PACS). There was no evidence of any change in the PACS score with time.

Daytime Functioning. We found that depressive symptoms, as measured by the QIDS, improved over time equally in both groups. There was no significant change in either StateTrait Anxiety Inventory (STAI)-trait scale, PCS, or MCS (SF-12) scores.

\section{Discussion}

The primary goal of this pilot study was to evaluate the potential efficacy of CBT-I in Veterans complaining of insomnia during early recovery from AD. We found that CBT-I improved insomnia more than a control treatment, effects that persisted after treatment ended. Treatment with CBT-I also improved the self-reported SL, decreased dysfunctional sleep-related cognitions, and improved sleep hygiene behaviors more than control. However, CBT-I was not superior to $\mathrm{MO}$ in improving PDA or daytime outcomes.

CBT-I exerted a large effect size on insomnia scores, consistent with those reported in prior studies (Currie et al., 2004, Arnedt et al., 2011). Thus, CBT-I may be beneficial in treating insomnia in alcohol dependent Veterans. The durability of improvement in insomnia after the 
end of CBT-I treatment has also been demonstrated in subjects with AD as well as in primary insomnia (Sivertsen et al., 2006, Currie et al., 2004).

Insomnia in $A D$ has been linked with cognitive distortions and dysfunctional beliefs about sleep (Brooks et al., 2016, Brower et al., 2001). Arnedt and colleagues reported an improvement in sleep-related cognitive distortions in a series of subjects treated with CBT-I (Arnedt et al., 2007). Our study extends this finding in the context of an RCT of CBT-I. Inadequate sleep hygiene-related behaviors such as daytime naps, which are commonly seen in this population, can perpetuate insomnia (Currie et al., 2003). We also extend the literature by demonstrating that CBT-I treatment differentially improved subjects' sleep hygiene scores.

Commonly reported insomnia symptoms include SL (difficulty falling asleep), NAW (multiple awakenings after falling asleep) and WASO (time spent awake after initially falling asleep). We showed a significant decrease in SL with CBT-I treatment, a finding that is consistent with two prior studies (Currie et al., 2004, Arnedt et al., 2011). However, in our study, CBT-I treatment was associated with a non-significant improvement in WASO, while in prior studies CBT-I resulted in significantly greater improvements in WASO. Prior studies have demonstrated that sustained abstinence from alcohol over time leads to improvements in objective sleep measures of sleep onset latency, slow wave sleep stages, and REM-sleep-related variables. However, fragmentation of sleep through the night may persist for up to two years into sobriety and may increase the risk of relapse (Drummond et al., 1998, Adamson and Burdick, 1973, Williams and Rundell, 1981). In our study, subjects were abstinent on only $27 \%$ of the days during the pretreatment period, whereas in the previous studies they were sober for an average of 2-4 months (Currie et al., 2004, Arnedt et al., 2011). Thus, it is possible that the persistent effects of alcohol on sleep maintenance were more evident in our subjects because they were still drinking at study entry. The small sample in our study may also have contributed to the non-significant effect on WASO.

The two prior RCTs reported no effect of CBT-I treatment on the number of subjects who relapsed to alcohol use or the number of days abstinent. Currie and colleagues found that during the 7-week treatment phase of their study, subjects in individual therapy consumed an 
average of 31.8 drinks on 10.3 drinking days, compared to 60 drinks on 9.7 drinking days in the wait-list control group. These numbers suggest that either more subjects in the control condition drank heavily or those who consumed alcohol drank heavily. This contrasts with the findings of Arnedt et al., where four subjects relapsed to heavy drinking in the CBT-I (N=9) and behavioral placebo $(\mathrm{N}=8)$ groups. However, Arnedt and colleagues used a very stringent criterion for relapse by considering all dropouts as having relapsed to drinking (Arnedt et al., 2011). In our study, 1 subject in the CBT-I and 2 in the MO groups relapsed to heavy drinking during the treatment phase. By the end of the post-treatment follow-up phase, three subjects in each arm had relapsed to heavy drinking. These preliminary data suggest that CBT-I treatment may not protect against relapse to drinking in recovering subjects. Lastly, the lack of differential improvement in the daytime functioning measures with CBT-I is similar to that reported in the prior two clinical trials.

A majority of Veterans who receive care at the Philadelphia CMCVAMC primary site identify themselves as African American. In addition to being located in Philadelphia, where the population is evenly divided between European Americans and African Americans, some prior studies have shown that African-American Veterans are more likely to be treated for substance use disorders than European-American Veterans (Glass et al., 2010, Williams et al., 2012). Therefore, it is not surprising that $73 \%$ of Veterans in this study identified themselves as African American. Because of the racial imbalance between the treatment arms, we treated race as a covariate in our statistical models. Race was an independent predictor of the change in percent days abstinent over time ( $\beta=-9.0,95 \%$ C.I.: $-9.7,-8.2)$, with African-American subjects reporting a decrease of $9 \%$ in the model-estimated mean PDA over time. It is possible that participation in the study bolstered the social support for these middle-aged, African-American subjects with co-occurring insomnia, leading to improved self-efficacy and consequently a relatively greater proportion of abstinent days, as shown previously (Warren et al., 2007, Walton et al., 2001). However, race was not a significant predictor of improvement in the sleep-related measures (ISI total score, sleep diary variables, DBAS or SHI), which could have been secondary to the small sample size that provided inadequate statistical power to detect a difference based on race or to evaluate psychosocial factors as mediators of racial differences. 
377 Although we replicated some findings from prior trials and report some novel findings, the selection of treatment-seeking, alcohol-dependent subjects introduced a selection bias so that these results may not generalize to all Veterans with AD. The effect sizes could have been skewed because of the decrease in sample size during the follow-up visits because of the dropouts. Furthermore, the sample size did not yield sufficient power to detect a difference in drinking outcomes between the treatment groups over time. Future studies should attempt to replicate these findings with a larger sample over a longer duration to provide adequate power to detect differences in drinking outcomes. Future studies should also use polysomnography to quantify objective sleep and spectral polysomnographic changes with treatment and evaluate the role of alcohol as a hypnotic agent.

\section{Conclusions.}

CBT-I was efficacious for treating insomnia in alcohol-dependent Veterans during early recovery. However, in this small RCT, it was not superior to the control group in improving abstinence from alcohol. Adequately-powered studies are required to evaluate the effect of CBT-I on drinking outcomes and to identify potential moderators of treatment response.

Registration and role of funding sources. clinicaltrials.gov identifier NCT01603381. The Competitive Pilot Project Fund of the VISN-4 VA provided funding for the conduct of this trial (SC). The study was funded by VA grant IK2CX000855 (to S.C.) and NIH grants R01 AG041783 (to M.L.P.), R56 AG050620 (to M.L.P.); and R01 AA023192 and R01 AA021164 (to H.R.K.).

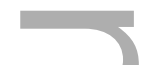

Contributors. SC, MLP and JTA conceptualized the study; SC and JF evaluated subjects; SC, KHM, JTA and MLP analyzed data; SC, KHM, JTA, MLP, HRK, DWO and JF collaborated in the generation of the final manuscript.

This article is protected by copyright. All rights reserved 
Conflict of interest. None of the authors report any conflict of interest with this investigation. Dr. Chakravorty has received research support from AstraZeneca and Teva pharmaceuticals. Dr. Perlis has received research support from Nexalin technology and Teva pharmaceuticals. Dr. Kranzler is a member of the American Society of Clinical Psychopharmacology's Clinical Trials Initiative (ACTIVE) group, which in the past three years was supported by AbbVie, Alkermes, Amygdala Neurosciences, Arbor, Ethypharm, Indivior, Lilly, Lundbeck, Otsuka, and Pfizer. He is named as an inventor on PCT patent application \#15/878,640 entitled: "Genotype-guided dosing of opioid agonists," filed January 24, 2018.

Acknowledgment: We thank Mr. Elliott Sturgis for assistance with the data collection. We also express our gratitude to Dr. Kachina Allen at Princeton University, who assisted in drafting the manuscript, Dr. Andrew Cucciara at the Perelman School of Medicine for his advice on the statistical analysis and the Translational Medicine and Applied Therapeutics of the Perelman School of Medicine for their support.

\section{References}

Adamson J, Burdick JA (1973) Sleep of dry alcoholics. Archives of general psychiatry 28:146-149. Alexander M, Ray MA, Hebert JR, Youngstedt SD, Zhang H, Steck SE, Bogan RK, Burch JB (2016) The National Veteran Sleep Disorder Study: Descriptive Epidemiology and Secular Trends, 2000-2010. Sleep 39:1399-1410.

Arnedt JT, Conroy D, Rutt J, Aloia MS, Brower KJ, Armitage R (2007) An open trial of cognitivebehavioral treatment for insomnia comorbid with alcohol dependence. Sleep medicine 8:176-180.

Arnedt JT, Conroy DA, Armitage R, Brower KJ (2011) Cognitive-behavioral therapy for insomnia in alcohol dependent patients: a randomized controlled pilot trial. Behav Res Ther 49:227-233. 
Barlow DH (1992) Cognitive-behavioral approaches to panic disorder and social phobia. Bull Menninger Clin 56:A14-28.

Bastien CH, Vallieres A, Morin CM (2001) Validation of the Insomnia Severity Index as an outcome measure for insomnia research. Sleep Med 2:297-307.

Blessed G, Tomlinson BE, Roth M (1968) The association between quantitative measures of dementia and of senile change in the cerebral grey matter of elderly subjects. Br J Psychiatry 114:797-811.

Bootzin DR, Perlis ML (2011) Stimulus Control Therapy, in A comprehensive primer of behavioral sleep medicine interventions, A comprehensive primer of behavioral sleep medicine interventions (PERLIS ML, ALOIA M, KUHN BR eds), pp 21-30, Elsevier Academic Press, Amsterdam.

Bootzin R (1972) Stimulus control treatment for insomnia, in Series Stimulus control treatment for insomnia, 80th Annual Convention, APA, pp 395-396.

Bootzin R (1984) Evaluation of stimulus control instructions, progressive muscle relaxation and sleep hygiene as treatments for insomnia. Sleep:142-144.

Brooks AT, Krumlauf M, Fryer CS, Beck KH, Yang L, Ramchandani VA, Wallen GR (2016) Critical Transitions: A Mixed Methods Examination of Sleep from Inpatient Alcohol Rehabilitation Treatment to the Community. PLoS One 11:e0161725.

Brower KJ, Aldrich MS, Robinson EA, Zucker RA, Greden JF (2001) Insomnia, self-medication, and relapse to alcoholism. Am J Psychiatry 158:399-404.

Chakravorty S, Chaudhary NS, Brower KJ (2016) Alcohol Dependence and Its Relationship With Insomnia and Other Sleep Disorders. Alcohol Clin Exp Res.

Currie SR, Clark S, Hodgins DC, El-Guebaly N (2004) Randomized controlled trial of brief cognitive-behavioural interventions for insomnia in recovering alcoholics. Addiction 99:1121-1132.

Currie SR, Clark S, Rimac S, Malhotra S (2003) Comprehensive assessment of insomnia in recovering alcoholics using daily sleep diaries and ambulatory monitoring. Alcoholism, clinical and experimental research 27:1262-1269. 
Drummond SP, Gillin JC, Smith TL, DeModena A (1998) The sleep of abstinent pure primary alcoholic patients: natural course and relationship to relapse. Alcoholism, clinical and experimental research 22:1796-1802.

Flannery BA, Volpicelli JR, Pettinati HM (1999) Psychometric properties of the Penn Alcohol Craving Scale. Alcoholism, clinical and experimental research 23:1289-1295.

Frank RH, Kaul JD (1978) The Hawthorne experiments: First statistical interpretation. Am Social Rev 43:623-643.

Friedmann PD, Rose JS, Swift R, Stout RL, Millman RP, Stein MD (2008) Trazodone for sleep disturbance after alcohol detoxification: a double-blind, placebo-controlled trial. Alcoholism, clinical and experimental research 32:1652-1660.

Glass JE, Perron BE, Ilgen MA, Chermack ST, Ratliff S, Zivin K (2010) Prevalence and correlates of specialty substance use disorder treatment for Department of Veterans Affairs Healthcare System patients with high alcohol consumption. Drug and alcohol dependence 112:150-155.

Johns MW (1991) A new method for measuring daytime sleepiness: the Epworth sleepiness scale. Sleep 14:540-545.

Jungquist CR, O'Brien C, Matteson-Rusby S, Smith MT, Pigeon WR, Xia Y, Lu N, Perlis ML (2010) The efficacy of cognitive-behavioral therapy for insomnia in patients with chronic pain. Sleep medicine 11:302-309.

Kelsall HL, Wijesinghe MS, Creamer MC, McKenzie DP, Forbes AB, Page MJ, Sim MR (2015) Alcohol use and substance use disorders in Gulf War, Afghanistan, and Iraq War veterans compared with nondeployed military personnel. Epidemiol Rev 37:38-54.

Martin JL, Schweizer CA, Hughes JM, Fung CH, Dzierzewski JM, Washington DL, Kramer BJ, Jouldjian S, Mitchell MN, Josephson KR, Alessi CA (2017) Estimated Prevalence of Insomnia among Women Veterans: Results of a Postal Survey. Womens Health Issues 27:366-373.

Mastin DF, Bryson J, Corwyn R (2006) Assessment of sleep hygiene using the Sleep Hygiene Index. Journal of behavioral medicine 29:223-227. 
McCarney R, Warner J, Iliffe S, van Haselen R, Griffin M, Fisher P (2007) The Hawthorne Effect: a randomised, controlled trial. BMC medical research methodology 7:30.

Morin CM, Belleville G, Belanger L, Ivers H (2011) The Insomnia Severity Index: psychometric indicators to detect insomnia cases and evaluate treatment response. Sleep 34:601-608.

Morin CM, Vallieres A, Ivers H (2007) Dysfunctional beliefs and attitudes about sleep (DBAS): validation of a brief version (DBAS-16). Sleep 30:1547-1554.

Perlis ML, Jungquist C, Smith MT, Posner D (2005) The Cognitive Behavioral Treatment of Insomnia: A Treatment Manual., Springer-Verlag.

Perlis ML, Smith MT, Orff H, Enright T, Nowakowski S, Jungquist C, Plotkin K (2004) The effects of modafinil and cognitive behavior therapy on sleep continuity in patients with primary insomnia. Sleep 27:715-725.

Posner D, Gehrman PR (2011) Sleep Hygiene., Academic Press, Amsterdam.

Rush AJ, Trivedi MH, Ibrahim HM, Carmody TJ, Arnow B, Klein DN, Markowitz JC, Ninan PT, Kornstein S, Manber R, Thase ME, Kocsis JH, Keller MB (2003) The 16-Item Quick Inventory of Depressive Symptomatology (QIDS), clinician rating (QIDS-C), and selfreport (QIDS-SR): a psychometric evaluation in patients with chronic major depression. Biol Psychiatry 54:573-583.

Seal KH, Cohen G, Waldrop A, Cohen BE, Maguen S, Ren L (2011) Substance use disorders in Iraq and Afghanistan veterans in VA healthcare, 2001-2010: Implications for screening, diagnosis and treatment. Drug and alcohol dependence 116:93-101.

Sheehan DV, Lecrubier Y, Sheehan KH, Amorim P, Janavs J, Weiller E, Hergueta T, Baker R, Dunbar GC (1998) The Mini-International Neuropsychiatric Interview (M.I.N.I.): the development and validation of a structured diagnostic psychiatric interview for DSM-IV and ICD-10. J Clin Psychiatry 59 Suppl 20:22-33;quiz 34-57.

Sivertsen B, Omvik S, Pallesen S, Bjorvatn B, Havik OE, Kvale G, Nielsen GH, Nordhus IH (2006) Cognitive behavioral therapy vs zopiclone for treatment of chronic primary insomnia in older adults: a randomized controlled trial. JAMA : the journal of the American Medical Association 295:2851-2858. 
Sobel LC, Sobell MB (1992) Timeline followback: a technique for assessing self-reported alcohol consumption. , Humana Press, New Jersey.

Spielberger CD, Gorsuch RL, Luchene R (1970) The state-trait anxiety inventory., Consulting Psychologist Press, Palo Alto, CA.

Spielman AJ, Caruso LS, Glovinsky PB (1987a) A behavioral perspective on insomnia treatment. Psychiatr Clin North Am 10:541-553.

Spielman AJ, Saskin P, Thorpy MJ (1987b) Treatment of chronic insomnia by restriction of time in bed. Sleep 10:45-56.

Stata Statistical Software: Release 12. [computer program]. College Station, TX: StataCorp LP; 2011.

Stepanski EJ, Wyatt JK (2003) Use of sleep hygiene in the treatment of insomnia. Sleep Med Rev 7:215-225.

Sullivan JT, Sykora K, Schneirderman J, Naranjo CA, Sellers EM (1989) Assessmnet of alcohol withdrawal: the revised clinical institute withdrawal assessment for alcohol scale. British Journal of Addiction 84:1353-1357.

Tsai J, Kasprow WJ, Rosenheck RA (2014) Alcohol and drug use disorders among homeless veterans: prevalence and association with supported housing outcomes. Addict Behav 39:455-460.

Research Randomizer (Version 3.0) [computer program]; 2013.

Walton MA, Blow FC, Booth BM (2001) Diversity in relapse prevention needs: gender and race comparisons among substance abuse treatment patients. The American journal of drug and alcohol abuse 27:225-240.

Ware JE, Kosinski M, Keller SD (1996) A 12-Item Short-Form Health Survey: Construction of Scales and Preliminary Tests of Reliability and Validity. Medical Care 34:220 - 233.

Warren JI, Stein JA, Grella CE (2007) Role of social support and self-efficacy in treatment outcomes among clients with co-occurring disorders. Drug and alcohol dependence 89:267-274.

Williams EC, Lapham GT, Hawkins EJ, Rubinsky AD, Morales LS, Young BA, Bradley KA (2012) Variation in documented care for unhealthy alcohol consumption across race/ethnicity 
in the Department of Veterans Affairs Healthcare System. Alcohol Clin Exp Res 36:16141622.

Williams HL, Rundell OH, Jr. (1981) Altered sleep physiology in chronic alcoholics: reversal with abstinence. Alcoholism, clinical and experimental research 5:318-325.

Zarcone VP (1989) Sleep Hygiene, in Principles and Practice of Sleep Medicine., Principles and Practice of Sleep Medicine. (KRYGER MH, ROTH T, DEMENT WC eds), W.B. Saunders Company, Philadelphia.

\section{Figure Legends}

Figure 2. Legend: $I S \mid=$ Insomnia Severity Index; $w k$ = week number of visit in the treatment phase; 6 mo. $=6$-month post-treatment follow-up visit; model statistics of mixed effects maximum likelihood regression using unstructured covariance matrix and adjusted for Race; time: $\beta=-1.05, p=0.01$; treatment: $\beta=1.5, p=0.5$; treatment $x$ time: $\beta=-1.3, p=0.03$;

Figure 3. Legend: SL = Sleep Latency (from their sleep diaries); $w k=$ week number of visit in the treatment phase; 6 mo. $=6$-month post-intervention follow-up visit; model statistics of mixed effects maximum likelihood regression using unstructured covariance matrix and adjusted for Race; time: $\beta=2.5, p=0.29$; treatment: $\beta=47.7, p=0.002$; treatment $x$ time: $\beta=1.5, p<0.0001$.

$$
\text { Figure 4. Legend: DBAS = Dysfunctional Attitudes and Beliefs about Sleep scale; wk = week }
$$
number of visit in the treatment phase; 6 mo. $=6$-month post-intervention follow-up visit; model statistics of mixed effects maximum likelihood regression using independent covariance matrix and adjusted for Race; time: $\beta=0.03, p=0.49$; treatment: $\beta=0.1, p=0.84$; treatment $x$ time: $\beta=-0.2, p<0.0001$ 
Table 1. Baseline demographic and clinical variables for the total sample and by treatment groups

\begin{tabular}{|c|c|c|c|}
\hline \multirow{2}{*}{ Variable } & \multirow{2}{*}{$\begin{array}{c}\text { Total Sample } \\
\text { mean (SD) } \\
(\mathrm{N}=22)\end{array}$} & \multicolumn{2}{|c|}{$\begin{array}{c}\text { Treatment groups } \\
\text { mean (SD) }\end{array}$} \\
\hline & & $\mathrm{MO}(\mathrm{N}=11)$ & CBT-I $(\mathrm{N}=11)$ \\
\hline \multicolumn{4}{|l|}{ Demographic } \\
\hline Age (yr) & 54.5 (6.9) & $56(6)$ & $52(7)$ \\
\hline Gender (male), N (\%) & $22(100 \%)$ & $11(100 \%)$ & $11(100 \%)$ \\
\hline Race (African American), N (\%) & $16(73 \%)$ & $11(100 \%)$ & $5(45 \%)^{*}$ \\
\hline Education (yr) & $12(0.8)$ & $12(0.9)$ & $12(0.8)$ \\
\hline Unemployed (past month), N (\%) & $14(64 \%)$ & $6(55 \%)$ & $8(73 \%)$ \\
\hline Marital Status (single), N (\%) & $18(82 \%)$ & $9(82 \%)$ & $9(82 \%)$ \\
\hline \multicolumn{4}{|l|}{ Sleep } \\
\hline Insomnia Severity Index total score & $18.9(5.4)$ & $19.8(5.6)$ & $18.0(5.3)$ \\
\hline Sleep Latency (sleep diary, min) & $51.4(50.6)$ & $33.9(20.3)$ & $69.0(65.5)$ \\
\hline Wake time after sleep onset (sleep diary, min) & $38.6(27.2)$ & $33.7(26.4)$ & $43.6(28.4)$ \\
\hline Total Sleep Time (sleep diary, min) & $286.4(98.8)$ & $283.1(115.3)$ & $289.6(84.7)$ \\
\hline Number of Awakenings (sleep diary, min) & $1.8(1.1)$ & $1.6(1.0)$ & $1.9(1.2)$ \\
\hline Dysfunctional Beliefs \& Attitudes mean score & $5.4(1.6)$ & $5.6(1.7)$ & $5.1(1.6)$ \\
\hline Sleep Hygiene Scale total score & $34.7(5.2)$ & $36.6(4.0)$ & $32.9(5.8)$ \\
\hline Epworth Sleepiness Scale total score & $11.1(5.2)$ & $13.1(5.6)$ & $9.1(4.1)$ \\
\hline \multicolumn{4}{|l|}{ Alcohol Consumption (TLFB) } \\
\hline Percent Days Abstinent & $26.4(26.3)$ & $25.4(30.9)$ & $27.4(22.3)$ \\
\hline Proportion of Heavy Drinking Days & $0.8(0.1)$ & $0.8(0.1)$ & $0.9(0.2)$ \\
\hline Penn Alcohol Craving Scale total score & $7.6(6.8)$ & $9.0(6.3)$ & $6.1(7.2)$ \\
\hline \multicolumn{4}{|l|}{ Daytime Dysfunction } \\
\hline Quick Inventory of Depressive Symptoms ${ }^{1}$ & $22.1(8.3)$ & $22.0(8.6)$ & $22.3(8.5)$ \\
\hline Trait Subscale (State Trait Anxiety Inventory) & $47.3(7.6)$ & $48.0(8.7)$ & $46.7(6.6)$ \\
\hline Physical Composite Score (SF-12 scale) & $39.6(4.6)$ & $39.3(4.0)$ & $40.0(5.3)$ \\
\hline Mental Composite Score (SF-12 scale) & $40.1(5.6)$ & $40.6(5.0)$ & $39.5(6.2)$ \\
\hline
\end{tabular}




\begin{tabular}{|l|c|c|c|}
\hline Hypnotic Medication Prescriptions (N=20) & & & \\
\hline Any medication for insomnia & $8(40 \%)$ & $3(30 \%)$ & $5(50 \%)$ \\
\hline Trazodone & $4(20 \%)$ & $3(30 \%)$ & $1(10 \%)$ \\
\hline Mirtazapine & $2(10 \%)$ & $0(0 \%)$ & $2(20 \%)$ \\
\hline Gabapentin & $1(5 \%)$ & $0(0 \%)$ & $1(10 \%)$ \\
\hline
\end{tabular}

$\mathrm{yr}=$ years; ${ }^{*} \mathrm{p}=0.01 ;{ }^{1}$ without the three sleep items

$\square$
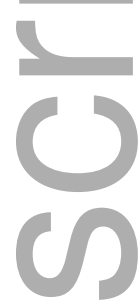

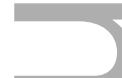
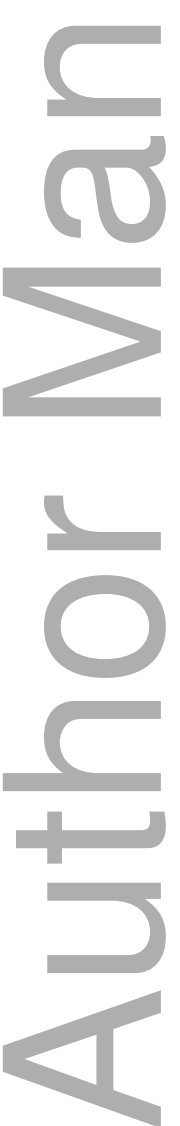

This article is protected by copyright. All rights reserved 
Table 2. Model-estimated mean change in clinical outcomes over time across treatment groups (change from baseline values)

\begin{tabular}{|c|c|c|c|c|c|c|c|c|c|c|c|c|}
\hline Variable & \multicolumn{4}{|c|}{ Change at end of treatment ${ }^{1}$} & \multicolumn{4}{|c|}{ Change at 3-month post-treatment follow-up ${ }^{1}$} & \multicolumn{4}{|c|}{ Change at 6-month post-treatment follow-up ${ }^{1}$} \\
\hline 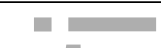 & MO & 95\% C.I. & CBT-I & 95\% C.I. & MO & 95\% C.I. & CBT-I & 95\% C.I. & MO & 95\% C.I. & CBT-I & 95\% C.I. \\
\hline \multicolumn{13}{|l|}{ Sleep } \\
\hline ISI total score & -8.4 & $-16.3,-0.5$ & -12.7 & $-15.5,-10.0$ & -9.4 & $-18.3,-0.5$ & -13.4 & $-16.0,-10.9$ & -10.5 & $-20.4,-0.6$ & -13.9 & $-16.3,-11.6$ \\
\hline SL (min) & 20.6 & $-18.2,59.5$ & -45.1 & $-78.0,-12.2$ & 23.2 & $-20.5,67.0$ & -36.8 & $-75.9,2.3$ & 25.8 & $-22.7,74.4$ & -25.4 & $-71.9,20.9$ \\
\hline WASO (min) & -1.7 & $-17.8,14.3$ & -24.5 & $-40.8,-8.3$ & -1.9 & $-20.1,16.1$ & -27.6 & $-45.9,-9.3$ & -2.1 & $-22.3,17.9$ & -30.7 & $-51.0,-10.4$ \\
\hline TST (min) & 45.2 & $0.8,89.6$ & 102.4 & $57.5,147.3$ & 50.9 & $0.9,100.8$ & 115.2 & $64.7,165.7$ & 56.5 & $1.0,112.0$ & 128.0 & $71.9,184.1$ \\
\hline NAW & -0.06 & $-1.0,0.8$ & -0.27 & $-1.2,0.6$ & -0.07 & $-1.1,0.9$ & -0.30 & $-1.3,0.7$ & -0.08 & $-1.2,1.0$ & -0.3 & $-1.5,0.8$ \\
\hline DBAS & 0.2 & $-0.5,1.1$ & $\begin{array}{l}-1.8 \\
\end{array}$ & $-2.6,-0.9$ & 0.3 & $-0.6,1.2$ & -2.0 & $-2.9,-1.1$ & 0.3 & $-0.6,1.3$ & -2.2 & $-3.2 .-1.2$ \\
\hline SHI & -0.8 & $-3.4,1.7$ & -4.6 & $-7.1,-2.0$ & -0.9 & $-3.8,1.9$ & -5.1 & $-8.0,-2.3$ & -1.0 & $-4.2,2.2$ & -5.7 & $-8.9,-2.5$ \\
\hline ESS & -0.6 & $-2.4,1.0$ & -3.1 & $-4.9,-1.4$ & -0.7 & $-2.7,1.1$ & -3.5 & $-5.5,-1.5$ & -0.8 & $-3.0,1.3$ & -3.9 & $-6.1,-1.7$ \\
\hline \multicolumn{13}{|l|}{ Alcohol } \\
\hline PDA & 0.7 & $0.6,0.8$ & 0.7 & $0.6,0.8$ & 0.7 & $0.6,0.8$ & 0.7 & $0.6,0.8$ & 0.7 & $0.6,0.8$ & 0.7 & $0.6,0.8$ \\
\hline
\end{tabular}

${ }^{1}$ As compared to the baseline phase; $\mathrm{MO}=$ Standard Care Monitor-Only condition; CBT-I = Cognitive Behavioral Therapy for Insomnia; ISI = Insomnia Severity Index total score; SL = Sleep Latency; $\min =$ minutes; WASO = Wake After Sleep Onset time; TST = Total Sleep Time; NAW = Number of Awakenings; DBAS = Dysfunctional Beliefs and Attitudes about Sleep Scale mean score; SHI = Sleep Hygiene Index total score; ESS = Epworth Sleepiness Scale total score; PDA = Percent Days Abstinent; PACS = Penn Alcohol Craving Scale total score; QIDS = 16-item Quick Inventory of Depressive Symptomatology; STAI-Trait = State Trait Anxiety Inventory - Trait subscale; PCS = Physical Composite Summary Score (from the Short Form 12-item measure); MCS = Mental Composite Summary Score (from the Short Form 12-item measure). The significant between-group differences over time are shown in bold.

This article is protected by copyright. All rights reserved 


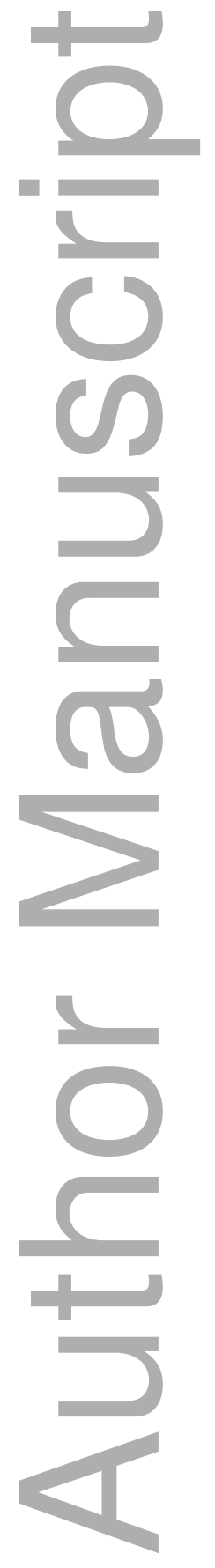

This article is protected by copyright. All rights reserved 


\section{Figure 1. CONSORT Flow Diagram of Subjects in the Study}

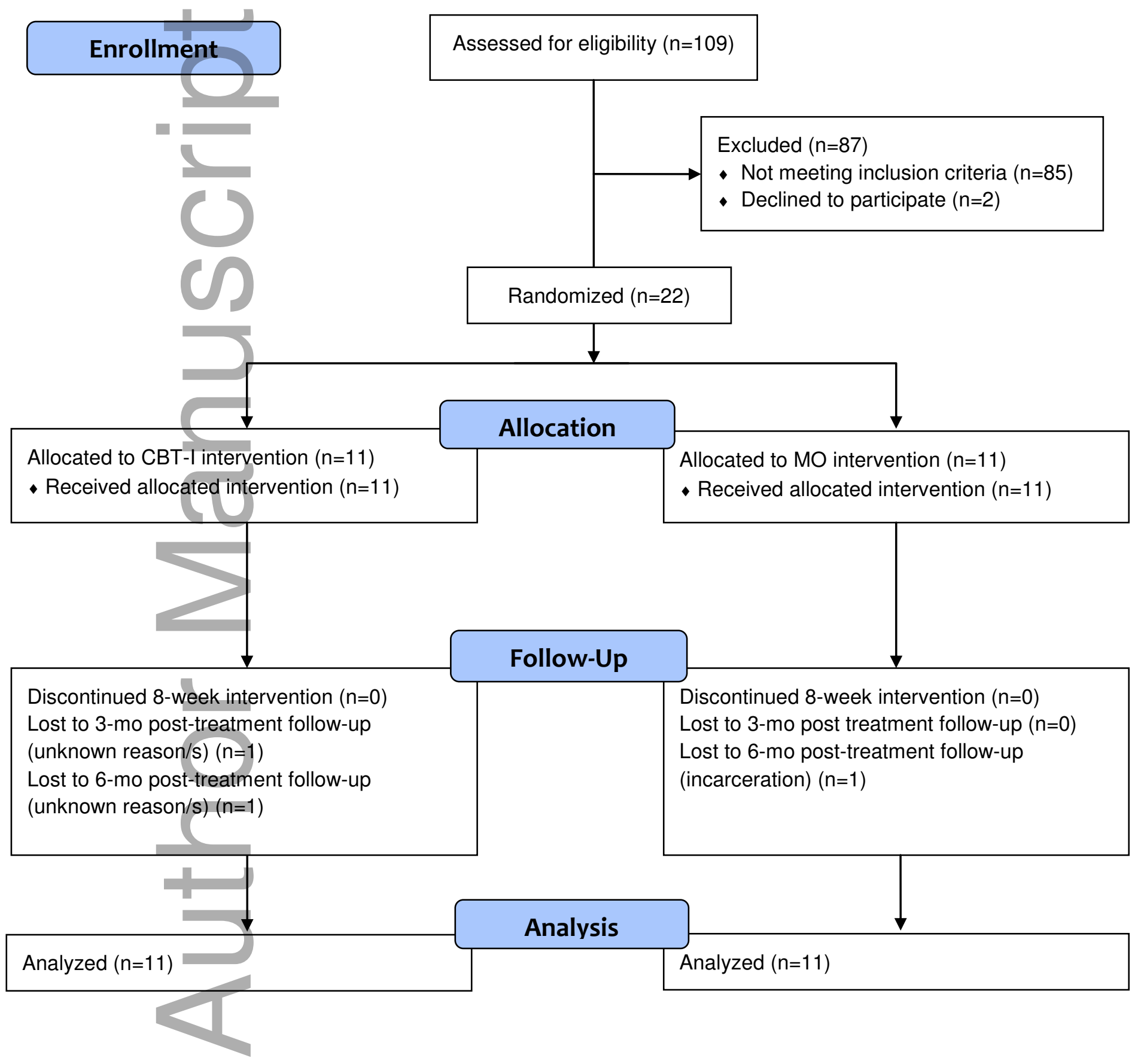

This article is protected by copyright. All rights reserved 
Figure 2. Model-estimated changes in Insomnia Severity Index (ISI) total score over time by treatment arm

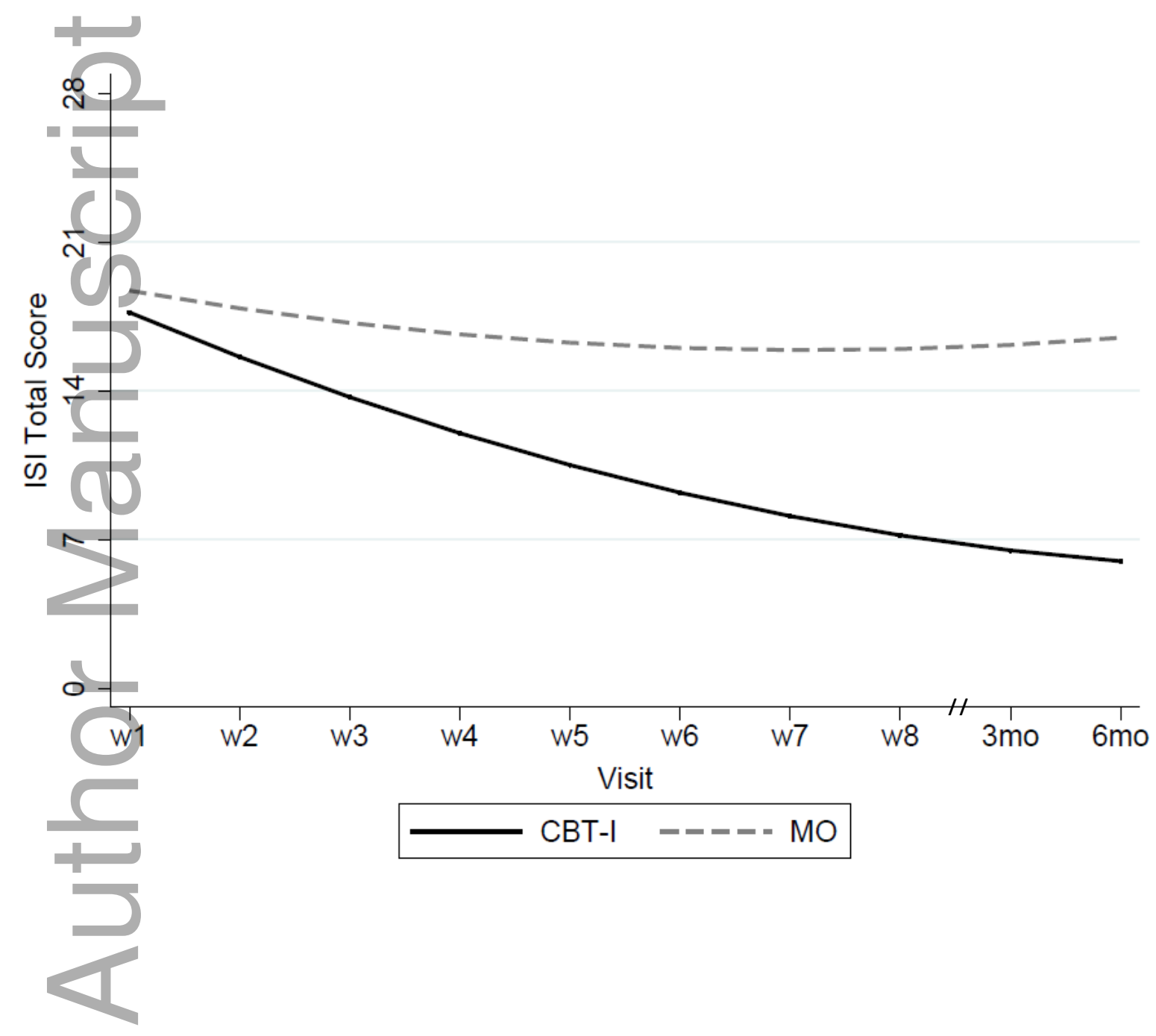

This article is protected by copyright. All rights reserved 
Figure 3. Model-estimated changes in subjective Sleep Latency (SL) over time by treatment arm

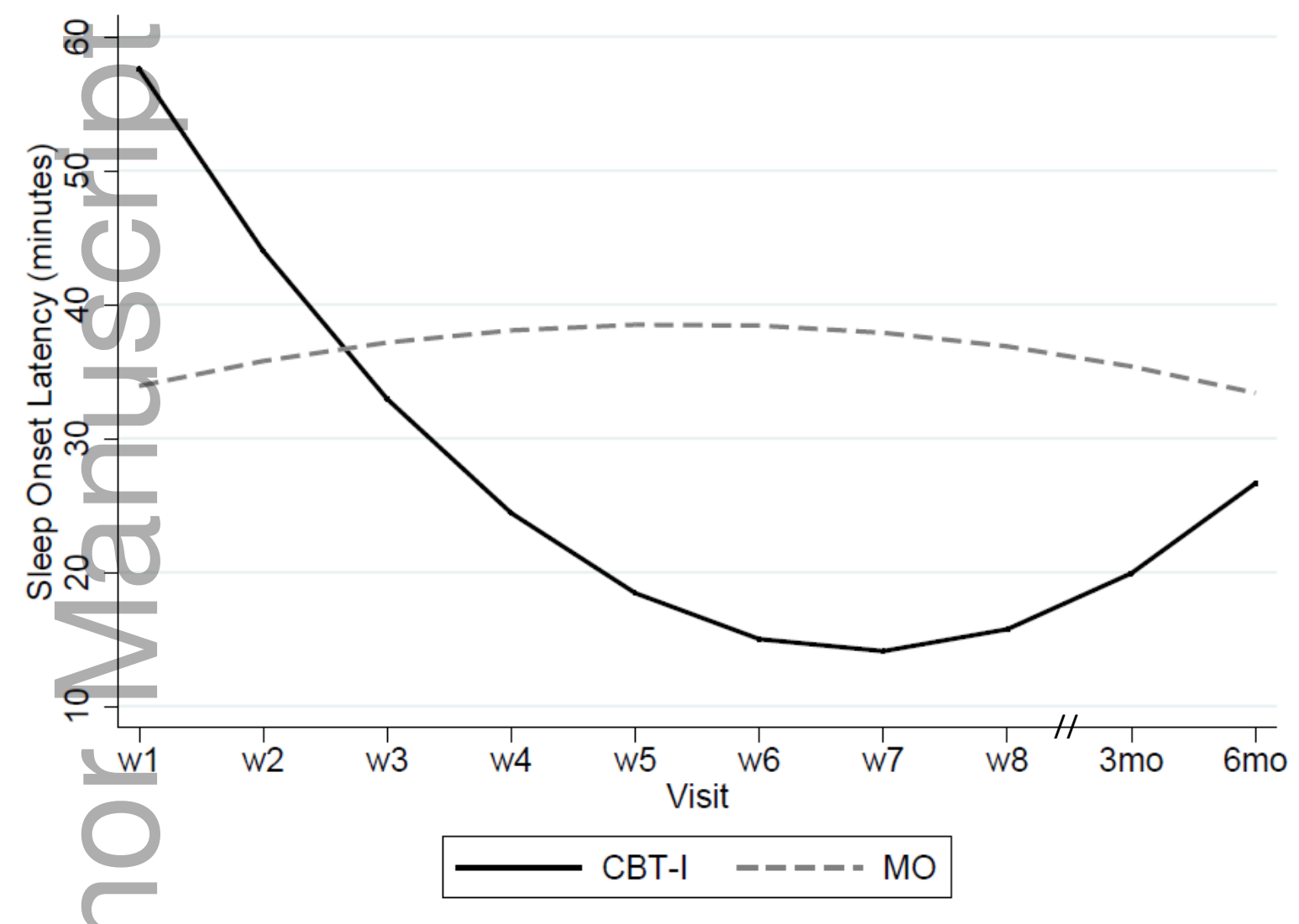

This article is protected by copyright. All rights reserved 
Figure 4. Model-estimated changes in Dysfunctional Beliefs and Attitudes About Sleep (DBAS) mean score over time

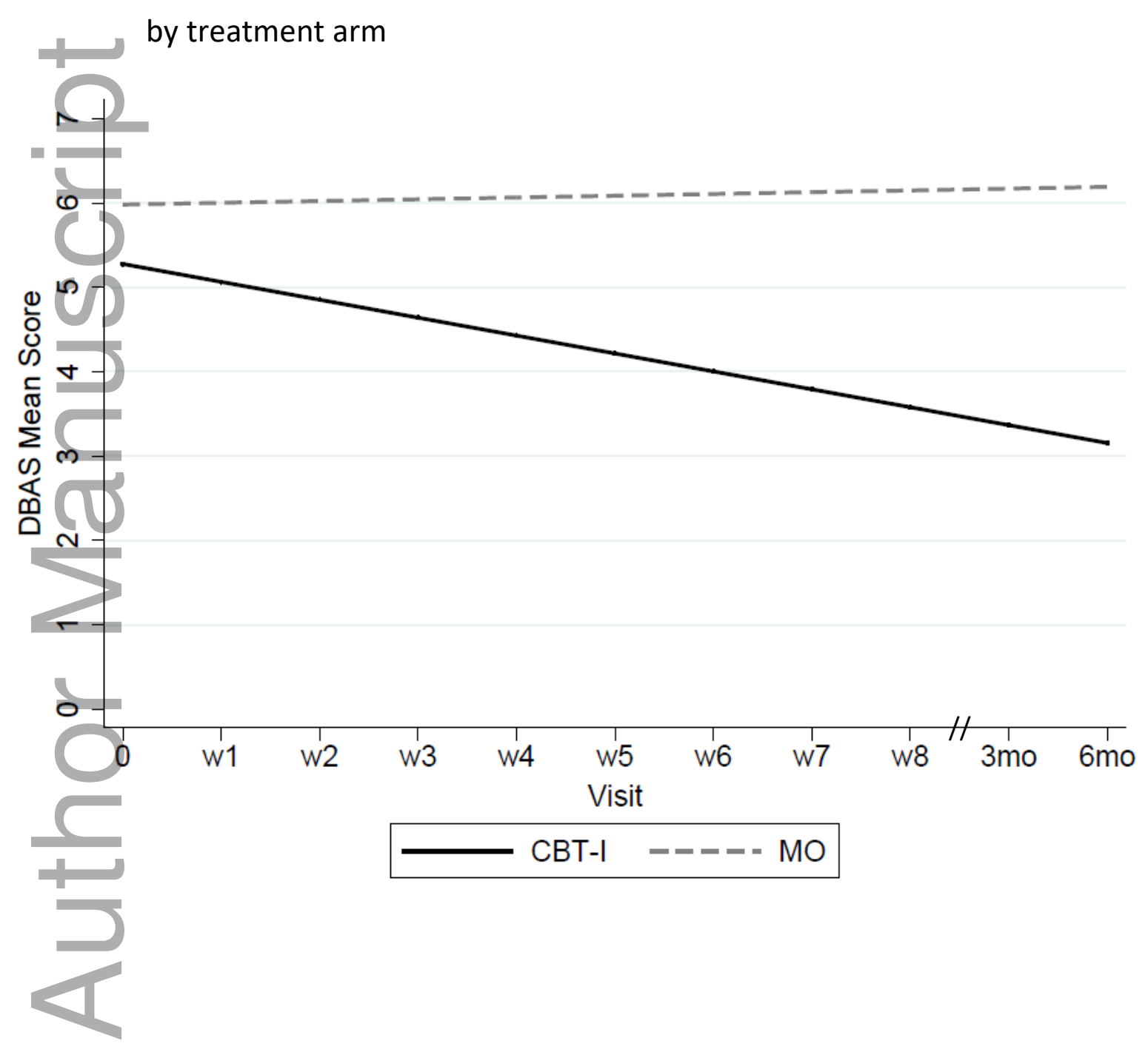

This article is protected by copyright. All rights reserved 